\title{
Detección de problemas de salud ocular en pacientes polimedicados a través de nuevos servicios profesionales en farmacia comunitaria
}

\author{
Luis Montalbán-Soler ${ }^{1,2}$, Mónica Ferrit-Martín ${ }^{3}$, Francisco José Fernández-Gómez ${ }^{1,2}$, Sandra Sierra-Alarcón ${ }^{4}$ \\ 1. Doctor en Farmacia. Área de Farmacia y Tecnología Farmacéutica, Departamento de Farmacología, Universidad de Murcia, Murcia, España. \\ 2. Farmacéutico comunitario (Murcia) 3. Doctora en Farmacia. Servicio de Inspección de Farmacia, Albacete, España. 4. Doctora en Farmacia. Colegio \\ Oficial de Farmacéuticos de la Región de Murcia (COFRM), Murcia, España.
}

\section{PALABRAS CLAVE}

Problemas de salud ocular, Servicios profesionales en farmacia comunitaria, paciente polimedicado

\section{ABREVIATURAS}

\section{AP: atención primaria} AV: agudeza visual $\mathrm{AV}_{\mathrm{CMC}}$ : agudeza visual con la mejor corrección posrefracción $\mathrm{AV}_{\mathrm{H}}$ : agudeza visual habitual CPM: conocimiento del paciente sobre su medicación ESVP: examen de salud visual protocolizado FC: farmacia comunitaria HTO: hipertensión ocular OMI: opacidad de los medios internos

OS: ojo seco

PIO: presión intraocular

PSO: problemas de salud ocular $\mathrm{PSO}_{\mathrm{C}}$ : PSO controlado

$\mathrm{PSO}_{\mathrm{NC}}$ : PSO no controlado $\mathrm{PSO}_{\mathrm{NC}}-\mathrm{C}: \mathrm{PSO}_{\mathrm{NC}}$ conocido $\mathrm{PSO}_{\mathrm{NC}}-\mathrm{NC}: \mathrm{PSO}_{\mathrm{NC}}$ no conocido SPESV: servicios profesionales especializados en salud visual

\section{KEYWORDS}

Ocular health problems, community pharmacy professional services, polymedicated patient

\section{RESUMEN}

Objetivo: analizar los resultados de un nuevo servicio profesional especializado en salud visual (SPESV) para la detección de problemas de salud ocular (PSO) en pacientes polimedicados e identificar posibles factores asociados a su aparición y control.

Métodos: estudio observacional y descriptivo. Emplazamiento: farmacia comunitaria en Murcia, España. Participantes: se incluyeron 92 pacientes que padecían al menos un PSO diagnosticado o sospecha y que utilizaban $\geq 4$ medicamentos crónicos. Mediciones principales: se realizó un examen de salud visual protocolizado (ESVP). Las variables dependientes fueron los tipos de PSO detectados en el ESVP y las independientes la adherencia y el conocimiento sobre el tratamiento crónico oftálmico, entre otras.

Resultados: el número total de PSO fue de 303 (3,3 PSO/paciente), de los que el $64 \%$ no estaba controlado ( $\mathrm{PSO}_{\mathrm{NC}}$ ) y el $32,3 \%$ era desconocido por el paciente. La mayor parte de PSO detectados fueron los relacionados con la refracción. El 57,1 \% de los pacientes en tratamiento con antiglaucomatosos y el $100 \%$ de los tratados para ojo seco denotaron una falta de adherencia al tratamiento; el 78,6\% y el 71,4\%, respectivamente, no conocían esta medicación. El análisis bivariante mostró que la edad, el nivel educativo y la profesión de los pacientes se relacionan con el número de $\mathrm{PSO}_{\mathrm{NC}}$.

Conclusiones: el SPESV en pacientes polimedicados permite la detección de un gran número de problemas de $\mathrm{PSO}_{\mathrm{NC}}$. Las principales intervenciones deben ir encaminadas a la mejorara de la adherencia y conocimiento de la medicación oftálmica, derivación al especialista y asesoramiento sobre la corrección oftálmica.

Detection of ocular health problems in polymedicated patients through new professional services in Community Pharmacy

\section{ABSTRACT}

Objective: Analyze the results of a new professional service specialized in visual health (PSSVH) for the detection of ocular health problems (OHP) in polymedicated patients and to identify possible factors associated with the appearance and control of these OHP.

Methods: Observational and descriptive study. Location: Community pharmacy in Murcia, Spain. Participants: 92 patients suffering at least from a diagnosed or suspected OHP and who used $\geq 4$ chronic medications were included. Main measurements: Patients were given a protocolised visual health examination (PVHE). The dependent variables were the types of OHP detected in the PVHE and the independent variables were adherence to and knowledge of chronic ophthalmic treatment among others.

Results: The total number of OHP was 303 (3.3 OHP/patient), of which 64\% were not controlled $\left(\mathrm{OHP}_{\mathrm{NC}}\right)$ and $32.3 \%$ were not known to the patient. Most of OHP detected were associated with refraction. 57,1\% of the patients undergoing treatment with antiglaucomatous drugs and 100\% of those treated for dry eye indicated non-adherence to treatment; 78.6\% and $71.4 \%$ respectively had no knowledge of this medication. The bivariate analysis showed that age, educational level and profession of the patients are related to the number of $\mathrm{OHP}_{\mathrm{NC}}$.

Conclusions: The PSSVH allows a large number of $\mathrm{OHP}_{\mathrm{NC}}$ in polymedicated patients. Main interventions should be aimed at improving adherence to and knowledge of ophthalmic medication, referrals to specialists and advice on ophthalmic correction.

Este artículo pertenece a la tesis doctoral "Evaluación e impacto de la implantación de nuevos Servicios Profesionales Especializados en Salud Visual en la Atención Farmacéutica al paciente crónico polimedicado", presentada y defendida en la Universidad de Murcia el 26 de julio de 2019, obra de D. Luis Montalbán Soler, farmacéutico comunitario en Águilas (Murcia). Dicho trabajo obtuvo el pasado 7 de noviembre durante las Jornada Nacional “SEFAC BILBA0 2019”, la segunda edición del Premio Francisco Martínez Romero a la mejor tesis doctoral en farmacia comunitaria asistencial, que promueve SEFAC en colaboración con eDUCO + Cofares Health.

Recibido: 8/1/2020

Aceptado: 7/4/2020

Disponible online: 11/5/2020
Financiación: ninguna.

Conflicto de intereses: ninguno.

Cite este artículo como: Montalbán-Soler L, Ferrit-Martin M, Fernández-Gómez FJ, Sierra-Alarcón S. Detección de problemas de salud ocular en pacientes polimedicados a través de nuevos servicios profesionales en farmacia comunitaria. Farmacéuticos Comunitarios. 2020 May 11:12(2):5-13 doi:10.33620/FC.2173-9218.(2020/Vol12).002.02

Correspondencia: Luis Montalbán-Soler (luis.montalban@um.es).

ISSN 1885-8619 @SEFAC (Sociedad Española de Farmacia Familiar y Comunitaria). Todos los derechos reservados. 


\section{Introducción}

Los problemas de salud ocular (PSO) relacionados con la edad tienen un elevado impacto social y económico, y se prevé que aumente en los próximos años debido al cada vez mayor envejecimiento de la población (1-3). Se han descrito diferentes causas por las que un gran número de personas mayores que padecen estos PSO los desconocen o no tienen ningún contacto directo con servicios especializados para su control (4) (5). Por todo esto, resulta prioritaria la creación y ofrecimiento de servicios de salud visual más accesibles a la población, orientados a la detección precoz y al control de los PSO (5-7).

La polimedicación, asociada a la edad y a la comorbilidad; aumenta el riesgo de que se produzca un conocimiento inapropiado sobre la medicación y una pérdida de adherencia terapéutica (8). En el caso concreto de algunas enfermedades oculares crónicas como el glaucoma y el ojo seco, la falta de adherencia al tratamiento supone un problema notable que puede afectar al control y prognosis de la enfermedad (3) (9) (10). Además, diversos estudios han demostrado la asociación de la comorbilidad con un mayor grado de deficiencia visual en pacientes mayores (11) (12). Por este motivo, numerosos trabajos publicados están dirigidos a la detección y control de PSO desde la Atención Primaria (AP) (6) (7) (13), siendo escasos los realizados desde la farmacia comunitaria (FC). Únicamente el estudio de Jiménez-Ramírez y cols. (14) señala al farmacéutico como un buen asesor de salud visual, aunque se precisa de una mayor evidencia científica.

Desde la FC se han desarrollado e implantado diferentes servicios profesionales orientados al paciente que han demostrado tener una influencia positiva sobre los objetivos terapéuticos y humanísticos en sujetos con enfermedades crónicas (15-17) y pacientes polimedicados (18). En este sentido, la cercanía y accesibilidad que ofrecen las FC en España y la confianza depositada por el paciente en el farmacéutico, hacen de la FC un lugar muy bien posicionado para la implantación de servicios profesionales especializados en salud visual (SPESV) dirigidos al paciente polimedicado.

\section{Objetivo}

El objetivo del presente estudio fue determinar el número y tipo de PSO detectados mediante un nuevo SPESV dirigido a pacientes polimedicados en una FC, así como identificar posibles factores asociados a la presencia y control de los PSO identificados.

\section{Material y métodos \\ Diseño del estudio}

Estudio observacional descriptivo realizado en una FC con servicios de óptica y optometría. El periodo de inclusión se realizó durante todo el mes de marzo de 2015.

\section{Población de estudio \\ y procedimiento}

Siguiendo los criterios de inclusión, la población de estudio fueron pacientes mayores de 18 años, polimedicados ( $\geq 4$ medicamentos prescritos durante al menos 3 meses) (19) (20) y que presentaban o tenían probabilidad de presentar al menos un PSO.

Los pacientes que cumplieron estos criterios fueron invitados a participar en el estudio por el personal de la farmacia; durante el proceso de dispensación de su medicación crónica. Durante este proceso, se realizó a cada paciente una anamnesis inicial sobre su historia médica oftalmológica, tratamientos, ayudas visuales y síntomas con el fin de seleccionar a aquellos pacientes con riesgo de presentar algún PSO no controlado ( $\mathrm{PSO}_{\mathrm{NC}}$ ), ya estuviese previamente diagnosticado o no (figura 1).

Todos los pacientes que aceptaron participar fueron sometidos a la realización de un examen de salud visual protocolizado (ESVP) con el fin de detectar PSO y determinar su control por parte del paciente a través de las medidas clínicas obtenidas. Tanto la realización del ESVP, como la recogida de datos fue siempre realizada por un mismo farmacéutico para eliminar la intervariabilidad, siendo además este facultativo graduado en Óptica y Optometría.

\section{Variables del estudio}

Las variables dependientes fueron los problemas de salud ocular (PSO). Dichos problemas, se definen según la WONCA como "cualquier queja, observación o hecho que el paciente y/ o el médico u otro profesional sanitario perciben como una desviación de la normalidad, que ha afectado, afecta, o puede afectar la capacidad funcional ocular del paciente" (21). Según los resultados obtenidos en el ESVP, los PSO se clasificaron como: 1) PSO controlado $\left(\mathrm{PSO}_{\mathrm{C}}\right)$ : diagnosticado previamente y cumple con la consecución de los objetivos clínicos o está bajo control de un especialista. 2) PSO no controlado $\left(\mathrm{PSO}_{\mathrm{NC}}\right)$ : no cumple con la consecución de los objetivos clínicos y sin control de un especialista. Podía ser conocido por el paciente por diagnóstico previo $\left(\mathrm{PSO}_{\mathrm{NC}}\right.$ conocido- $\left.\mathrm{PSO}_{\mathrm{Nc}}-\mathrm{C}\right)$ o no $\left(\mathrm{PSO}_{\mathrm{NC}}\right.$ no conocido-PSO $\mathrm{NC}-\mathrm{NC}$ ) (figura 1).

Las variables independientes del estudio fueron: edad, sexo, país de origen, nivel de estudios, profesión, número de medicamentos empleados, número de problemas de salud general, adherencia y conocimiento del paciente sobre su medicación (CPM) crónica oftálmica.

La medida de la adherencia se realizó mediante el cuestionario Simplified Medication Adherence Questionnaire (SMAQ) (22). El conocimiento del paciente sobre su medicación (CPM) fue evaluado mediante un test validado por García-Delgado y cols. en 2009 (23). Tanto para la medida de la adherencia como para el CPM se seleccionó tan sólo un medicamento por patología ocular elegido de manera aleatoria. Con el fin de asegurar la correcta administración de gotas o pomadas oftálmicas, en todos los casos de pacientes que estaban en tratamiento con alguna de estas formas farmacéutica, se aportó información oral y escrita sobre su correcta forma de administración, reforzándola de forma activa en cada una de las dispensaciones que se realizaron tras el estudio.

\section{Examen de salud visual protocolizado y clasificación} de los PSO

Este examen se basó en el estudio de seis puntos principales: 1) Medida de la refracción: se utilizaron un autorrefractómetro (Nidek AR-600 ${ }^{\circledR}$ ) y un foróptero (Nidek RT-2100 ${ }^{\circledR}$ ) para medir el tipo de ametropía y la corrección óptica necesaria para alcanzar la mejor agudeza visual (AV). 


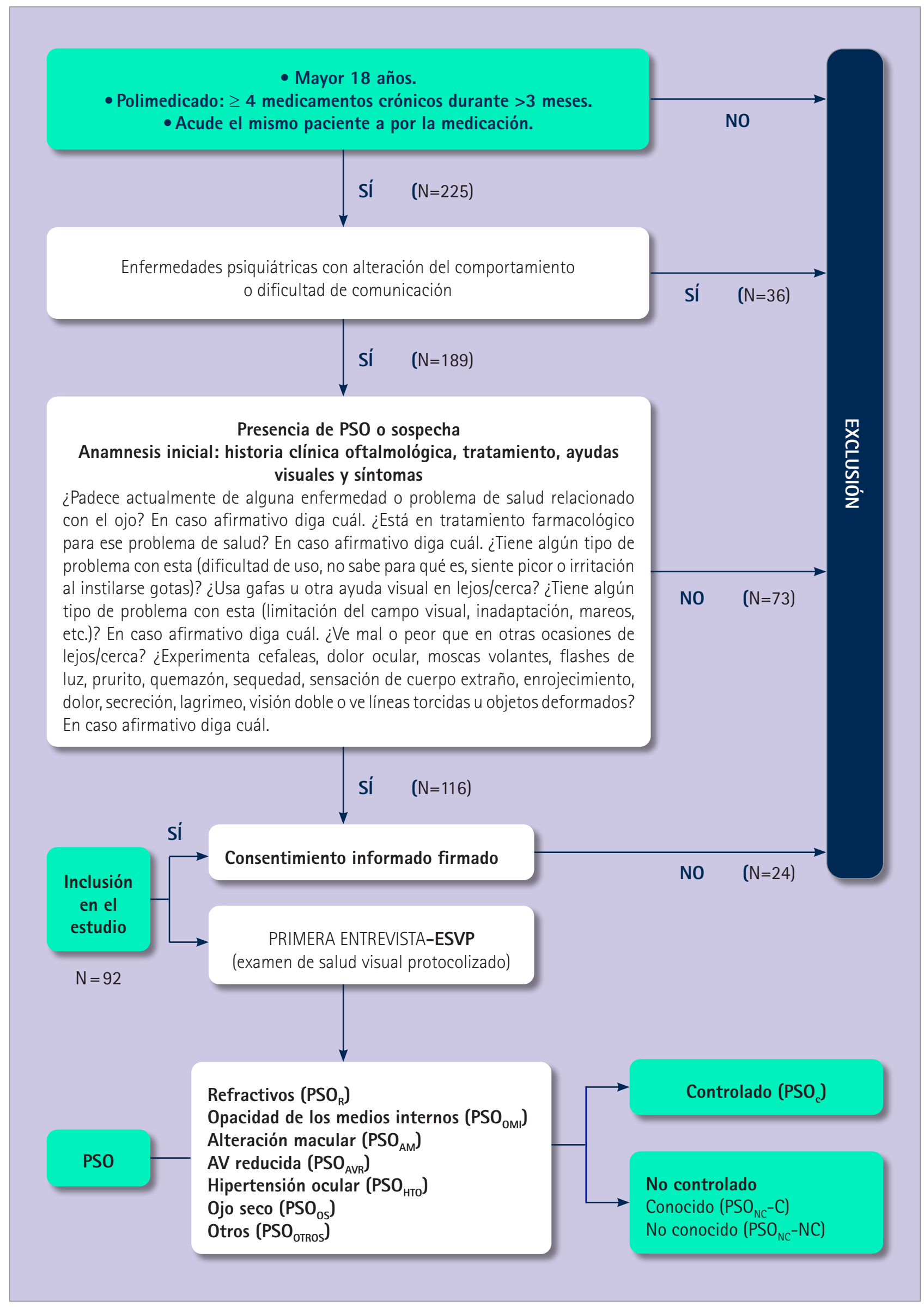

PSO: problema de salud ocular.

Figura 1 Diseño del estudio y algoritmo de detección de problemas de salud ocular (PSO) para la inclusión en el estudio 
2) Medida de la AV: se utilizaron optotipos de Snellen en escala decimal para medir la AV con la corrección habitual empleada por el paciente (agudeza visual habitual, $\mathrm{AV}_{\mathrm{H}}$ ); y la AV con su mejor corrección tras la refracción (agudeza visual con la mejor corrección posrefracción, $\mathrm{AV}_{\mathrm{CMC}}$ ) en condiciones de mono/binocularidad para visión lejana y en binocular para visión cercana. 3) Biomicroscopía del polo anterior: se utilizó una lámpara de hendidura (Slitlamp microscope SM- ${ }^{\circledR}$ ) para la detección de opacidades de los medios internos (OMI)/ cataratas que comprometan la $\mathrm{AV}_{\text {смс }}$ en al menos un ojo por debajo de los valores considerados de normali$\left.\operatorname{dad}\left(\mathrm{AV}_{\text {СмС }}<1,0\right)(4)(24) .4\right)$ Test de la rejilla de Amsler: se empleó para la detección precoz de síntomas relacionados con alteraciones maculares de la retina (25). 5) Medida de la presión intraocular (PIO): se utilizó un tonómetro de no-contacto computarizado (Topcon CT-80 ${ }^{\circledR}$ ), tomando como valor de la PIO la media de tres medidas para cada ojo. Se consideró un valor de PIO >21 mmHg como elevado (4) (26). 6) Test clínicos para ojo seco (OS): se realizaron tres pruebas para el diagnóstico de OS: a) Test de Schirmer I, evalúa la producción acuosa de lágrima, valores $<10 \mathrm{~mm}$ indicaron un resultado alterado (27). b) Fluorescein tear break-up time (TBUT), evalúa la estabilidad lagrimal, tiempos de ruptura de la lágrima $<10$ segundos indicaron un resultado alterado (27). c) Dry Eye Questionnaire (DEQ), 2001 (28), se compone de 4 items que recogen la frecuencia de los principales síntomas de OS; (a) disconfort y sequedad; b) sensación cuerpo extraño; c) ardor y picor; d) cansancio y pérdida de calidad de visión. No existe un criterio establecido para el diagnóstico de OS (27) (28) por lo que se consideraron pacientes con OS no controlado a aquellos que refirieron al menos un síntoma de forma frecuente o constante en el DEQ, acompañado de al menos un signo (Schirmer o TBUT alterados).

Como se puede observar en la figura 1; los PSO detectados se clasificaron en función de las pruebas que componen el ESVP en: 1) refractivos $\left(\mathrm{PSO}_{\mathrm{R}}\right)$; 2) opacidad de los medios internos $\left.\left(\mathrm{PSO}_{\mathrm{OMI}}\right) ; 3\right)$ alteración macular $\left(\mathrm{PSO}_{\mathrm{AM}}\right)$; 4) $\mathrm{AV}$ reducida $\left(\mathrm{PSO}_{\mathrm{AVR}}\right)$ no justificada por un $\mathrm{PSO}_{\mathrm{R}}, \mathrm{PSO}_{\mathrm{OMI}}$ o un $\mathrm{PSO}_{\mathrm{AM}}$; 5) hipertensión ocular $\left(\mathrm{PSO}_{\text {нто }}\right.$; 6) ojo seco $\left(\mathrm{PSO}_{\mathrm{OS}}\right)$, y 7 ) otros $\left(\mathrm{PSO}_{\text {отRos }}\right)$, derivados de otros PSO no contemplados en el ESVP (conjuntivitis, miodesopsias, problemas con el uso de gafas, etc.).

\section{Análisis estadístico}

Las variables numéricas se describen como media y desviación estándar y las categóricas como frecuencias y porcentaje. La normalidad de las variables se contrastó con el test de Kolmogorov-Smirnov. Para estudiar la relación entre el número de PSO detectados y el resto de variables, se realizó un análisis bivariante, empleando el test $t$ de Student y ANOVA. En los casos en los que no se cumplió la normalidad se empleó el test de Mann-Whitney y Kruskal-Wallis. Para el análisis bivariante el nivel de significación se estableció en $\mathrm{p}<0,05$. El software utilizado fue el SPSS ${ }^{\circledR}$, versión 19.

\section{Aspectos éticos}

En todos los casos se garantizó la confidencialidad de los datos acorde al cumplimiento de la Ley de protección de datos de carácter personal (Ley orgánica 15/1999). El protocolo de investigación fue aprobado por la Comisión de Ética en Investigación de la Universidad de Granada y todos los pacientes del estudio firmaron el consentimiento informado.

\section{Resultados}

Se seleccionaron 116 pacientes que cumplieron los criterios de inclusión y se consideraron un 20,7\% de pérdidas, resultando una población final de 92 pacientes (figura 1). Las características generales de la población se muestran en la tabla 1. La media de edad fue de $66,4 \pm 12,2$ años y el 58,7\% de los pacientes tenía $\geq 65$ años. Fundamentalmente fueron mujeres (70,7 \%), de nacionalidad española (96,7\%), amas de casa, jubiladas o en paro $(64,1 \%)$, sin estudios $(43,5 \%)$ o con estudios primarios (46.7\%). La media de problemas de salud general/paciente fue de $6.5 \pm 2.5$, siendo el promedio de medicamentos empleados/paciente de $9,1 \pm 4$, de los cuales el 36,9 \% empleaba al menos un medicamento de uso oftálmico (tabla 1).

En relación a las patologías, el $15,2 \%(n=14)$ de los pacientes estaba en tratamiento oftálmico para glaucoma/hipertensión ocular (HTO), de los
Tabla 1 Caracteristicas generales de la muestra $(n=92)$

\begin{tabular}{|c|c|c|}
\hline & $\mathrm{n}$ & $(\%)$ \\
\hline \multicolumn{3}{|l|}{ Sexo } \\
\hline Hombre & 27 & $(29,3)$ \\
\hline Mujer & 65 & $(70,7)$ \\
\hline \multicolumn{3}{|l|}{ Edad } \\
\hline $34-54$ & 17 & $(18,5)$ \\
\hline $55-64$ & 21 & $(22,8)$ \\
\hline $65-74$ & 21 & $(22,8)$ \\
\hline $75-86$ & 33 & $(35,9)$ \\
\hline \multicolumn{3}{|l|}{ Pais de origen } \\
\hline España & 89 & $(96,7)$ \\
\hline Otros & 3 & $(3,3)$ \\
\hline \multicolumn{3}{|l|}{ Profesión } \\
\hline $\begin{array}{l}\text { Ama de casa/Jubilado/ } \\
\text { Paro }\end{array}$ & 59 & $(64,1)$ \\
\hline $\begin{array}{l}\text { Trabajos manuales no } \\
\text { cuaificados }\end{array}$ & 15 & $(16,3)$ \\
\hline $\begin{array}{l}\text { Trabajos manuales } \\
\text { cualificados }\end{array}$ & 9 & $(9,8)$ \\
\hline $\begin{array}{l}\text { Administrativos y } \\
\text { comerciales }\end{array}$ & 5 & $(5,4)$ \\
\hline $\begin{array}{l}\text { Clase dirigente y } \\
\text { empresarios }\end{array}$ & 4 & $(4,3)$ \\
\hline \multicolumn{3}{|l|}{ Nivel de estudios } \\
\hline Sin estudios & 40 & $(43,5)$ \\
\hline Estudios primarios & 43 & $(46,7)$ \\
\hline Estudios secundarios & 9 & $(9,8)$ \\
\hline Estudios universitarios & 0 & $(0)$ \\
\hline \multicolumn{3}{|c|}{ Problemas de salud general } \\
\hline$\leq 5$ & 34 & $(37,0)$ \\
\hline $6-7$ & 29 & $(31,5)$ \\
\hline$>7$ & 29 & $(31,5)$ \\
\hline \multicolumn{3}{|c|}{ Número medicamentos uso general } \\
\hline $4-5$ & 23 & $(25,0)$ \\
\hline $6-10$ & 34 & $(37,0)$ \\
\hline$>10$ & 35 & $(38,0)$ \\
\hline \multicolumn{3}{|c|}{ Número medicamentos uso oftálmico } \\
\hline 0 & 58 & $(63,1)$ \\
\hline 1 & 20 & $(21,7)$ \\
\hline$>1$ & 14 & $(15,2)$ \\
\hline \multicolumn{3}{|c|}{$\begin{array}{l}\text { Adherencia y CPM crónica oftálmica } \\
\text { glaucoma/HTO }(n=14)\end{array}$} \\
\hline Adherente & 6 & 42,9 \\
\hline No adherente & 8 & 57,1 \\
\hline Conoce & 3 & 21,4 \\
\hline No conoce & 11 & 78,6 \\
\hline \multicolumn{3}{|c|}{$\begin{array}{l}\text { Adherencia y CPM crónica oftálmica ojo } \\
\text { seco }(n=21)\end{array}$} \\
\hline Adherente & 0 & 0 \\
\hline No adherente & 21 & 100 \\
\hline Conoce & 6 & 28,6 \\
\hline No conoce & 15 & 71,4 \\
\hline
\end{tabular}

HTO: hipertensión ocular. CPM: conocimiento del paciente sobre su medicación. 
Tabla 2 Caracteristicas clínicas reportadas del ESVP de los pacientes incluidos en el estudio ( $n=92)$

\begin{tabular}{|c|c|c|c|c|c|}
\hline & $\mathrm{n}$ & $\%$ & & $\mathrm{n}$ & $\%$ \\
\hline \multicolumn{3}{|l|}{ Refracción } & \multicolumn{3}{|l|}{ Test ojo seco } \\
\hline \multicolumn{3}{|l|}{ Ametropía tras refracción } & \multicolumn{3}{|l|}{ Signos de ojo seco (pruebas objetivas) } \\
\hline Emétrope & 7 & 7,6 & TBUT+ & 27 & 29 \\
\hline Miope & 15 & 16,3 & Schirmer+ & 8 & 8,7 \\
\hline Hipermétrope & 62 & 67,4 & TBUT-Schirmer+ & 40 & 43,5 \\
\hline Miope/Hipermétrope ${ }^{a}$ & 8 & 8,7 & Total & 75 & 81,5 \\
\hline Astigmatismo & 88 & 95,7 & Síntomas de ojo seco & & \\
\hline Presbicia & 87 & 94,6 & Disconfort y sequedad & & \\
\hline \multicolumn{3}{|l|}{ Medida de la AV (visión lejana) } & (0) Nunca & 8 & 8,7 \\
\hline \multicolumn{3}{|l|}{$\mathrm{AV}_{\mathrm{H}} / \mathrm{AV}_{\mathrm{CMC}}$ binocular } & (1) Raramente & 21 & 22,8 \\
\hline$\geq 1,0$ (normal) & $38 / 60$ & $41,3 / 65,2$ & (2) A veces & 24 & 26,1 \\
\hline$<1,0->0,5$ (casi normal) & $39 / 26$ & $42,4 / 28,3$ & (3) Frecuentemente & 14 & 15,2 \\
\hline$\leq 0,5->0,1$ (deficiencia visual) & $15 / 6$ & $16,3 / 6,5$ & (4) Constantemente & 25 & 27,2 \\
\hline$\leq 0,1$ (ceguera legal) & $0 / 0$ & 0/0 & \multicolumn{3}{|l|}{ Sensación cuerpo extraño } \\
\hline \multicolumn{3}{|l|}{$\mathrm{AV}_{\mathrm{H}} / \mathrm{AV}_{\mathrm{CMC}}$ monocular $^{\mathrm{b}}$} & (0) Nunca & 24 & 26,1 \\
\hline$\geq 1,0$ (normal) & $25 / 43$ & $27,2 / 46,7$ & (1) Raramente & 33 & 35,9 \\
\hline$<1,0->0,5$ (casi normal) & $32 / 34$ & $34,8 / 37,0$ & (2) A veces & 27 & 29,3 \\
\hline$\leq 0,5->0,1$ (deficiencia visual) & $30 / 11$ & $32,6 / 12,0$ & (3) Frecuentemente & 7 & 7,6 \\
\hline$\leq 0,1$ (ceguera legal) & $5 / 4$ & $5,4 / 4,3$ & (4) Constantemente & 1 & 1,1 \\
\hline \multicolumn{3}{|l|}{ Biomicroscopía } & \multicolumn{3}{|l|}{ Ardor y picor } \\
\hline $\mathrm{OMI}$ con $\mathrm{AV}<1,0$ en un ojo & 12 & 13,0 & (0) Nunca & 24 & 26,1 \\
\hline $\mathrm{OMI}$ con $\mathrm{AV}<1,0$ en los dos ojos & 15 & 16,3 & (1) Raramente & 28 & 30,4 \\
\hline Total & 27 & 29,3 & (2) A veces & 28 & 30,4 \\
\hline \multicolumn{3}{|l|}{ Test rejilla Amsler } & (3) Frecuentemente & 7 & 7,6 \\
\hline Test + en un ojo & 7 & 7,6 & (4) Constantemente & 5 & 5,4 \\
\hline Test + en los dos ojos & 4 & 4,3 & \multicolumn{3}{|l|}{ Cansancio y pérdida calidad de visión } \\
\hline Total + & 11 & 12,0 & (0) Nunca & 19 & 20,7 \\
\hline \multicolumn{3}{|l|}{ Medida de la PIO } & (1) Raramente & 21 & 22,8 \\
\hline $\mathrm{PIO}>21 \mathrm{mmHg}$ en un ojo & 7 & 7,60 & (2) A veces & 28 & 30,4 \\
\hline $\mathrm{PIO}>21 \mathrm{mmHg}$ en los dos ojos & 5 & 5,4 & (3) Frecuentemente & 17 & 18,5 \\
\hline Total PIO >21 mmHg & 12 & 13,0 & (4) Constantemente & 7 & 7,6 \\
\hline
\end{tabular}

${ }^{a}$ Miopia en un ojo e hipermetropia en el otro.

${ }^{b} \mathrm{AV}$ monocular para el peor ojo de cada paciente.

$\mathrm{AV}_{\mathrm{H}}$ : agudeza visual habitual/AV $\mathrm{CMC}$ : agudeza visual con la mejor corrección posrefracción.

que el 57,1\% no era adherente y el $78,6 \%$ no tenía un conocimiento adecuado. Además, el 22,8 \% $(n=21)$ de los individuos estaba en tratamiento oftálmico para OS, de los que el $100 \%$ no era adherente y el $71,4 \%$ no tenía un conocimiento adecuado (tabla 1).

La tabla 2 muestra los resultados del ESVP. El 16,3 \% y el $38 \%$ de los pacientes presentaban deficiencia visual $\left(\mathrm{AV}_{\mathrm{H}} \leq 0,5\right)$ antes del ESVP en visión binocular y monocular (ojo con peor $\mathrm{AV})$, respectivamente; reduciéndose estos porcentajes al 6,5\% en binocular y al 16,3\% en monocular tras la refracción. Por otra parte, en el 29,3\% de los pacientes se observó alguna opacidad de los medios internos acompañada de $\mathrm{AV}_{\mathrm{CMC}}<1,0$. El $12 \%$ dio positivo al test de la rejilla de Amsler y el $13 \%$ presentó un valor de $\mathrm{PIO}>21 \mathrm{mmHg}$ en al menos un ojo. Los síntomas de ojo seco referidos de forma frecuente $o$ constante siguieron el siguiente orden de frecuencia: disconfort y sequedad $(\mathrm{n}=39)$, cansancio y pérdida de calidad de visión $(n=24)$, ardor y picor $(n=12)$ y sensación de cuerpo extraño $(n=8)$. El 44,6\% refirió padecer al menos un síntoma de forma frecuente/constante; acompañado en el $95 \%$ de los casos de, al menos, un signo (tabla 2).

El número total de PSO fue de 303 (3,3 PSO/paciente); el $64 \%$ no estaba controlado $\left(\mathrm{PSO}_{\mathrm{NC}}\right)$, de los cuales el 50,5\% era desconocido por el paciente $\left(\mathrm{PSO}_{\mathrm{NC}}-\mathrm{NC}\right)$. En la tabla 3 se indica el número de sujetos y porcentaje de los tipos de PSO detectados en el ESVP, en función de su control y conocimiento previo por parte del paciente. De forma resumida, en el histograma de la figura 2 se ilustra la proporción de los distintos tipos de PSO en relación a los PSO totales.

Finalmente, se observó una relación estadísticamente significativa entre la edad ( $p=0,001)$, el país de origen $(p=0,014)$ y el nivel de estudios $(\mathrm{p}=0,012)$ y el promedio de PSO/paciente y entre la edad ( $\mathrm{p}=0.009)$, profesión $(p=0.010)$ y nivel de estudios $(\mathrm{p}=0.012)$ con el promedio de $\mathrm{PSO}_{\mathrm{NC}} /$ paciente (tabla 4). 
Tabla 3 Tipos de PSO detectados en el ESVP basándose en su control y conocimiento previo por parte del paciente

\begin{tabular}{|c|c|c|c|c|c|c|c|c|c|c|}
\hline & \multicolumn{2}{|c|}{$\mathrm{PSO}_{\mathrm{C}}$} & \multicolumn{2}{|c|}{$\mathrm{PSO}_{\mathrm{NC}-} \mathrm{C}$} & \multicolumn{2}{|c|}{$\mathrm{PSO}_{\mathrm{NC}-} \mathrm{NC}$} & \multicolumn{2}{|c|}{$\mathrm{PSO}_{\mathrm{NC}-\mathrm{TOTAL}}$} & \multicolumn{2}{|c|}{$\mathrm{PSO}_{\text {TOTAL }}$} \\
\hline & $n$ & (\%) & $\mathrm{n}$ & $(\%)$ & $n$ & (\%) & $n$ & & $n$ & $(\%)$ \\
\hline PSO-R lejos & 38 & $(34,9)$ & 24 & (25) & 21 & $(21,4)$ & 45 & $(23,2)$ & 83 & $(27,4)$ \\
\hline PSO-R cerca & 33 & $(30,3)$ & 41 & $(42,7)$ & 10 & $(10,2)$ & 51 & $(26,3)$ & 84 & $(27,7)$ \\
\hline PSO-OMI & 6 & $(5,5)$ & 4 & $(4,2)$ & 17 & $(17,3)$ & 21 & $(10,8)$ & 27 & $(8,9)$ \\
\hline PSO-AM & 6 & $(5,5)$ & 0 & (0) & 5 & $(5,1)$ & 5 & $(2,6)$ & 11 & $(3,6)$ \\
\hline PSO-AVR & 0 & (0) & 0 & (0) & 3 & $(3,1)$ & 3 & $(1,5)$ & 3 & $(1,0)$ \\
\hline PSO-HTO & 8 & $(7,3)$ & 6 & $(6,2)$ & 6 & $(6,1)$ & 12 & $(6,2)$ & 20 & $(6,6)$ \\
\hline PSO-OS & 9 & $(8,3)$ & 13 & $(13,5)$ & 26 & $(26,5)$ & 39 & $(20,1)$ & 48 & $(15,8)$ \\
\hline PSO-OTROS & 9 & $(8,3)$ & 8 & $(8,3)$ & 10 & $(10,2)$ & 18 & $(9,3)$ & 27 & $(8,9)$ \\
\hline TOTAL & 109 & (100) & 96 & (100) & 98 & (100) & 194 & (100) & 303 & (100) \\
\hline
\end{tabular}

PSO-R lejos: refracción visión lejana. PSO-R cerca: refracción visión cercana. PSO-OMI: opacidad de los medios internos con AV<1,0. PSO-AM: signos de alteración macular. PSO-AVR: agudeza visual reducida no justificada. PSO-HTO: hipertensión ocular. PSO-OS: ojo seco. PSO-OTROS: otros PSO no contemplados en el ESVP. PSO_: PSO controlados. PSO $\mathrm{NC}_{\mathrm{NC}}$-C: PSO no controlados, conocidos por el paciente. PSO $\mathrm{NC}_{\mathrm{NC}}$-NC: PSO no controlados, no conocidos por el paciente. PSO $\mathrm{NC}^{- \text {torata }}$ : PSO no controlados totales $\left(\mathrm{PSO}_{\mathrm{NC}}-\mathrm{C}+\mathrm{PSO}_{\mathrm{NC}}-\mathrm{NC}\right)$. $\mathrm{PSO}_{\text {TOTAL }}:$ : $\mathrm{PSO}_{\mathrm{C}}+\mathrm{PSO}_{\mathrm{NC}} \mathrm{TOTOAL}^{-}$

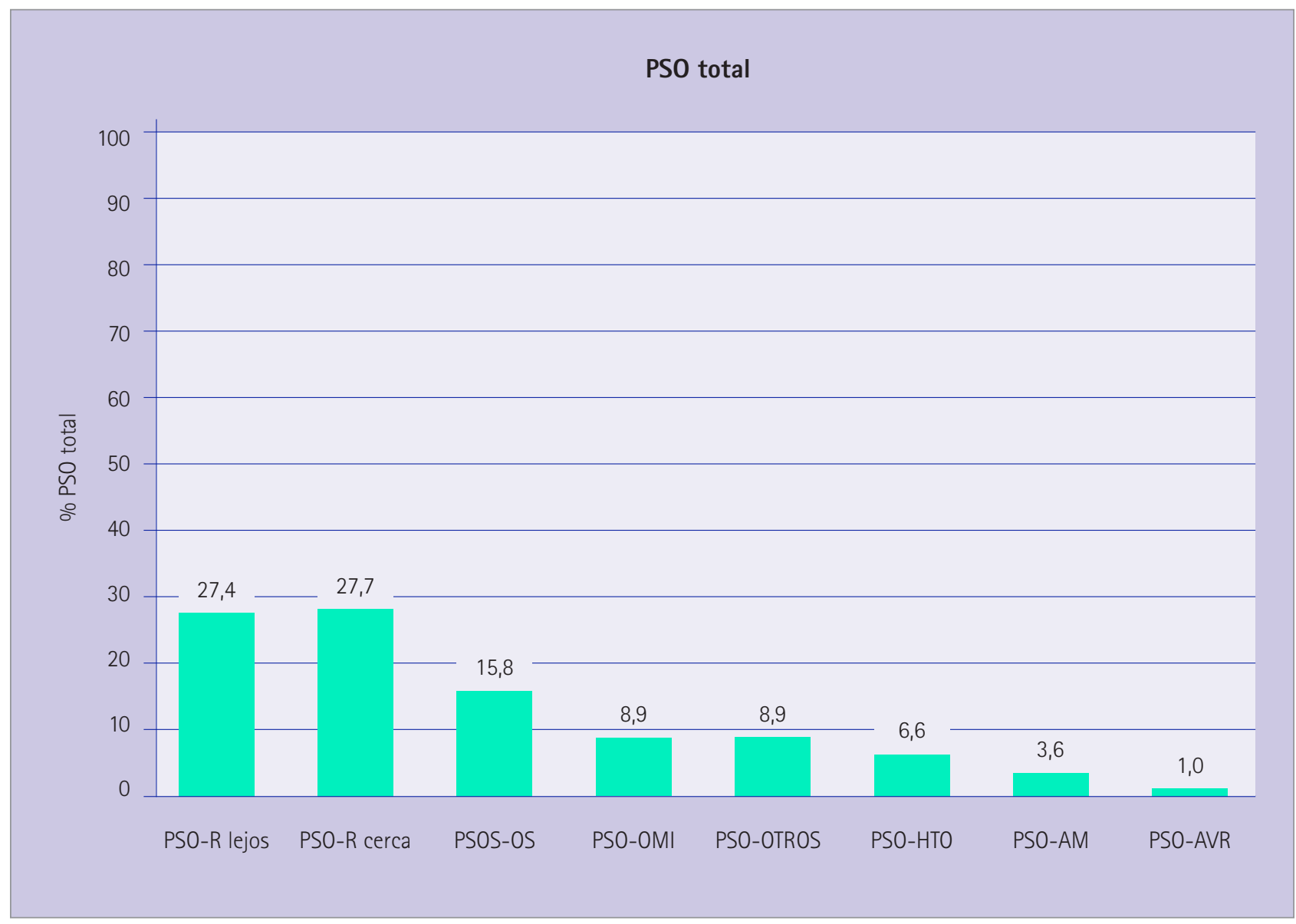

PSO-R lejos: refracción visión lejana (27,4\%). PSO-R cerca: refracción visión cercana (27,7 \%). PSO-0S: ojo seco (15,8 \%). PSO-0MI: opacidad de los medios internos con AV<1,0 (8,9\%). PSO-OTROS: otros PSO no contemplados en el ESVP (8,9\%). PSO-HTO: hipertensión ocular (6,6 \%). PSO-AM: signos de alteración macular (3,6 \%). PSOAVR: agudeza visual reducida no justificada (1\%).

Figura 2 Tipos de PSO detectados en relación a número total de PSO de la muestra 
Tabla 4 Relación de las variables que caracterizan a la muestra con el número de PSO por paciente

\begin{tabular}{|c|c|c|c|c|}
\hline & $\begin{array}{l}\mathrm{PSO}_{\text {TOTAL }} / \text { Paciente } \\
\text { (Media } \pm \mathrm{DE})\end{array}$ & $\mathrm{p}^{\mathrm{a}}$ & $\begin{array}{l}\text { PSO }_{\mathrm{NC}} / \text { Paciente } \\
\text { (Media } \pm \mathrm{DE})\end{array}$ & $p^{b}$ \\
\hline Sexo & & 0,105 & & 0,069 \\
\hline Hombre & $2,96 \pm 1,25$ & & $1,66 \pm 1,04$ & \\
\hline Mujer & $3,42 \pm 1,17$ & & $2,16 \pm 1,22$ & \\
\hline $\mathrm{Edad}^{c}$ & & 0,001 & & 0,009 \\
\hline$<67$ años & $2,84 \pm 1,17$ & & $1,68 \pm 1,03$ & \\
\hline$\geq 67$ años & $3,70 \pm 1,10$ & & $2,33 \pm 1,24$ & \\
\hline Pais de origen & & 0,014 & & 0,315 \\
\hline España & $3,34 \pm 1,19$ & & $2,03 \pm 1,19$ & \\
\hline Otros & $1,67 \pm 0,58$ & & $1,33 \pm 0,58$ & \\
\hline Profesión & & 0,116 & & 0,010 \\
\hline $\begin{array}{l}\text { Ama/o de casa, jubilada/o, } \\
\text { en paro }\end{array}$ & $3,54 \pm 1,15$ & & $2,27 \pm 1,18$ & \\
\hline $\begin{array}{l}\text { Trabajo manual no } \\
\text { cualificado }\end{array}$ & $2,93 \pm 1,16$ & & $2 \pm 1,13$ & \\
\hline Trabajo manual cualificado & $2,67 \pm 1,5$ & & $1,22 \pm 0,97$ & \\
\hline Administrativo o comercial & $2,40 \pm 0,89$ & & $1,5 \pm 1$ & \\
\hline Dirigente o empresaria/o & $3,25 \pm 0,96$ & & - & \\
\hline Estudios & & 0,012 & & 0,012 \\
\hline Sin estudios & $3,7 \pm 1,14$ & & $2,41 \pm 1,18$ & \\
\hline Estudios primarios & $2,88 \pm 1,22$ & & $1,73 \pm 1,14$ & \\
\hline Estudios secundarios & $3,33 \pm 0,87$ & & $1,55 \pm 0,88$ & \\
\hline Estudios universitarios & 0 & & 0 & \\
\hline Problemas de salud generalc & & 0,570 & & 0,422 \\
\hline$\leq 6$ & $3,57 \pm 1,146$ & & $1,91 \pm 1,18$ & \\
\hline$>6$ & $3,72 \pm 1,22$ & & $2,11 \pm 1,18$ & \\
\hline $\begin{array}{l}\text { Número de medicamentos } \\
\text { totalc }\end{array}$ & & 0,176 & & 0,525 \\
\hline$\leq 9$ & $3,41 \pm 1,04$ & & $1,94 \pm 1,26$ & \\
\hline$>9$ & $3,91 \pm 1,23$ & & $2,1 \pm 1,08$ & \\
\hline $\begin{array}{l}\text { Número de medicamentos } \\
\text { oftálmicos }\end{array}$ & & 0,766 & & 0,397 \\
\hline 0 & $3,17 \pm 1,15$ & & $2,09 \pm 1,13$ & \\
\hline 1 & $4,18 \pm 0,87$ & & $2,15 \pm 1,11$ & \\
\hline$>1$ & $4,28 \pm 1,04$ & & $1,78 \pm 1,37$ & \\
\hline
\end{tabular}

a Test Mann-Whitney y Kruskal-Wallis.

- Test $t$ Student o ANOVA en los casos en los que se cumple la normalidad. Test de Mann-Whitney y KruskallWallis en los no normales.

' La edad, los problemas de salud general y el número de medicamentos total se han categorizado en función de la mediana de la muestra.

$\mathrm{PSO}_{\text {TOTAL }} /$ Paciente: promedio del número de PSO por paciente. $\mathrm{PSO}_{\mathrm{NC}} /$ Paciente: promedio del número de PSO $_{\mathrm{NC}}$ por paciente. DE: desviación estándar.

\section{Discusión}

Según los resultados obtenidos, aproximadamente $2 / 3$ de los PSO detectados en el ESVP no estaban controlados, y la mitad de estos eran desconocidos por parte del propio paciente. Diferentes estudios realizados en pacientes mayores indican que esta falta de control y conocimiento sobre los PSO podría estar relacionada con factores como la falta de concluyendo que el $70 \%$ de la población necesitaba al menos uno de estos servicios y más del $30 \%$ necesitaba más de uno (5). Los servicios más necesarios en función de los PSO detectados fueron los relacionados con la refracción, seguidos de revisiones periódicas de la visión y servicios de cirugía de cataratas (5). En nuestro estudio los $\mathrm{PSO}_{\mathrm{R}}$ resultaron ser los más comunes y peor controlados, seguidos de $\mathrm{PSO}_{\mathrm{OS}}$ y $\mathrm{PSO}_{\text {OMI }}$ (tabla 3).

Tras el examen refractivo se determinó que el número de pacientes con deficiencia visual $\left(\mathrm{AV}_{\mathrm{H}} \leq 0,5\right)$ podría reducirse en más de la mitad mediante el empleo o cambio de la corrección óptica empleada, propiciando una mejora sustancial de la función visual de la muestra. Del mismo modo sucede con los pacientes con $\mathrm{PSO}_{\text {OMI }}$ no controlados $(n=21)$, en los que, de confirmarse el diagnóstico de cataratas por un especialista, éstas podrían ser operadas con la consecuente mejora de $\mathrm{AV}$ y de la calidad de vida relacionada con la salud (24).

A pesar de que existe cierta controversia sobre la efectividad de los screenings para la detección de glaucoma basados en la medida de la PIO, el valor estadístico poblacional de HTO establecido (PIO $\geq 21 \mathrm{mmHg}$ ) se acepta por la mayoría de autores como motivo de derivación en AP (13) (26). En nuestro estudio, la mitad de pacientes que presentaron una PIO elevada $(\mathrm{n}=6)\left(\mathrm{PSO}_{\mathrm{NC}}\right)$ no estaba en tratamiento ni bajo el control de un especialista $\left(\mathrm{PSO}_{\mathrm{NC}}-\mathrm{NC}\right)$ (tabla 3 ). En estos casos, la intervención del farmacéutico mediante derivación podría resultar fundamental en el diagnóstico precoz y pronóstico de una enfermedad silenciosa como es el glaucoma.

En nuestro trabajo se detectó una alta prevalencia de pacientes que refirieron tener algún sintoma relacionado con OS de forma frecuente/ constante acompañado de, al menos, un signo clínico $(42,4 \%)\left(\mathrm{PSO}_{\mathrm{NC}}\right)$, de los cuales el 66,6\% no estaba en tratamiento $\left(\mathrm{PSO}_{\mathrm{NC}}-\mathrm{NC}\right.$ ) (tabla 3). El OS representa el mayor porcentaje de motivo de consulta en Oftalmología. Sin embargo, en el $60 \%$ de los casos sus manifestaciones son leves. La puesta en marcha de acciones a nivel primario para su detección y control podría contribuir en la mejora de la eficiencia del sistema sanitario (27). 
En lo referente a pacientes en tratamiento con antiglaucomatosos, el $57,1 \%$ denotó una falta de adherencia al tratamiento de los cuales sólo el $25 \%$ alcanzó el objetivo terapéutico (PIO $\leq 21 \mathrm{mmHg}$ ) (26), mientras que el $100 \%$ de los pacientes adherentes lo alcanzó. Esto indica una posible incidencia de la adherencia sobre el control de la enfermedad (9) (10). En cuanto al CPM crónica oftálmica en glaucoma y OS, el 78,6 \% y el 71,4\%, respectivamente, no conocía de forma satisfactoria esta medicación, por lo que resulta imprescindible reforzar el grado de conocimiento y la adherencia en estos pacientes (tabla 1).

El análisis bivariante no mostró relación entre el número de PSO por paciente y otros dos factores estrechamente asociados a la polimedicación, como son el número de problemas de salud $(p=0.570)$ y los medicamentos utilizados $(p=0.176)$. No obstante, sí lo hizo con la edad $(\mathrm{p}=0,001)$ y el nivel de estudios $(p=0,012)$ (tabla 4). De igual forma, la falta de conocimiento y adherencia a la medicación crónica oftálmica podría deberse, junto con la edad, al bajo nivel educativo de la muestra (43,5 \% sin estudios).

Diferentes fármacos pueden producir, en muy diferente grado, efectos negativos sobre las diferentes estructuras oculares. Así, por ejemplo, el uso de antiagregantes plaquetarios o anticoagulantes (ácido acetilsalicílico o acenocumarol) puede producir hemorragia subconjuntival, los antipalúdicos (cloroquina o hidroxicloroquina) y antinoplásicos (tamoxifeno) aparición de retinopatías, los fármacos para la disfunción eréctil (sildenafilo), cianopsia o visión con coloración azulada, los retinoides (isotretinoina) y antidepresivos tricíclicos (amitriptilina) sequedad ocular, los antiepilépticos (topiramato) glaucoma de ángulo estrecho, y los corticoides (prednisona o dexametasona) aumento de la PIO y formación de cataratas (29) (30). Como hemos visto, en nuestro estudio el análisis de la relación entre los PSO y la medicación utilizada por los pacientes se hizo de forma cuantitativa y no cualitativa (por grupo terapéutico), no encontrándose estudios similares con los que comparar. Sin embargo, la alta prevalencia de pacientes con síntomas o signos de OS de nuestra muestra podría estar relacionada con el elevado uso de fármacos como antidepresivos, ansiolíticos, antihipertensivos o antialérgicos, los cuales pueden producir alteraciones de la película lagrimal o reducción de la secreción de lágrima (27) (29) (30). En este sentido, los servicios de atención farmacéutica prestados por el farmacéutico pueden ayudar a prevenir o reducir los resultados negativos de la medicación y sus posibles efectos sobre el ojo y la visión.

La implantación y realización de estos servicios supondrán, lógicamente, una inversión para la farmacia y un coste para el paciente. Sin embargo, consideramos que, a medio-largo plazo, esta inversión se verá rentabilizada con creces por su valor asistencial y una mayor fidelización de nuestros pacientes. No se ha realizado una estimación exacta del coste que supone la prestación de los SPESV, pero teniendo en cuenta diferentes factores como: a) tiempo empleado para la entrevista y ESVP (30-40 minutos por paciente), b) salario base de un farmacéutico comunitario, y c) coste de adquisición del aparataje empleado, estimamos que la retribución de este servicio para que sea sostenible en el tiempo debería situarse en torno a los 45-50 euros por visita (como muestra de gratitud de su participación en el estudio, ninguno de los pacientes abonó ningún tipo de honorario durante el servicio).

Desafortunadamente, los servicios de óptica y optometría y personal capacitado no son muy frecuentes en la FC, por lo que este factor puede ser una limitación para la implantación de estos servicios en otras FC. Sin embargo, estos resultados representan una contribución importante hacia un nuevo paradigma de atención farmacéutica en salud visual desde la AP, que podría contribuir al ahorro del coste indirecto de utilización de recursos sanitarios y del gasto farmacéutico, con la consecuente mejora de la eficiencia y sostenibilidad del Sistema Nacional de Salud (18).

\section{Conclusiones}

La implantación de SPESV en FC revela una alta prevalencia de PSO no controlados en pacientes polimedicados que afectan a la calidad de vida relacionada con la salud, poniendo de manifiesto la necesidad del servicio. Los principales factores asociados a los PSO y a su falta de control fueron la edad avanzada y el bajo nivel de estudios. La intervención farmacéutica debe ir encaminada en estos pacientes a la mejora de la adherencia y conocimiento sobre la medicación crónica oftálmica, así como a la derivación al especialista y recomendaciones sobre el uso de lentes oftálmicas en los casos en los que sea necesario, con el fin de mejorar el control de estos PSO detectados.

\section{Agradecimientos}

Este artículo forma parte de la tesis doctoral de D. Luis Montalbán Soler, dentro del Programa de Doctorado en Integración y Modulación de Señales en Biomedicina de la Universidad de Murcia, España. Queremos dar un especial agradecimiento a todo el equipo humano y personal de la Farmacia Ldo. José López Gil y a Dña. Manuela Expósito Ruiz, así como al profesor D. Manuel Canteras Jordana, por su ayuda con el análisis estadístico de los resultados.

\section{Referencias bibliográficas}

1. Pascolini D, Mariotti SP. Global estimates of visual impairment: 2010. Br J Ophthalmol. 2012;96:614-8. doi:10.1136/ bjophthalmol-2011-300539.

2. Congdon N, O'Colmain B, Klaver CC, Klein R, Muñoz B, Friedman DS, et al. Eye Diseases Prevalence Research Group. Causes and prevalence of visual impairment among adults in the United States. Arch Ophthalmol. 2004;122: 477-85. doi:10.1001/archo pht.122.4.477

3. Cardarelli WJ, Smith RA. Managed care implications of age-related ocular conditions. Am J Manag Care. 2013;19(Suppl 5):S85-91.

4. Reidy A, Minassian DC, Vafidis G, Joseph J, Farrow S, Wu J, et al. Prevalence of serious eye disease and visual impairment in a north London population: population based, cross sectional study. BMJ. 1998;316:16436. doi:10.1136/bmj.316.7145.1643

5. Zheng Y, Cheng CY, Lamoureux EL, Chiang PP, Rahman Anuar A, Wang JJ, et al. How much eye care services do Asian populations need? Projection from the Singapore Epidemiology of Eye Disease (SEED) study. Invest Ophthalmol Vis Sci. 2013;54:2171-7. doi:10.1167/iovs.12-11393

6. Ramke J, Brian G, Naduvilath T. Refractive error and presbyopia in $\mathrm{Ti}$ mor-Leste: the impact of 5 years of 
a national spectacle program. Invest Ophthalmol Vis Sci. 2012;53:434-9. doi:10.1167/iovs.11-8161

7. Chou R, Dana T, Bougatsos C. Screening older adults for impaired visual acuity: a review of the evidence for the U.S. Preventive Services Task Force. Ann Intern Med. 2009;151:44-58. doi:10.7326/0003-4819-151-1-20090 7070-00008

8. Mannucci PM, Nobili A; REPOSI Investigators. Multimorbidity and polypharmacy in the elderly: lessons from REPOSI. Intern Emerg Med. 2014;9:723-34. doi:10.1007/s11739-014-1124-1

9. Akpek EK, Smith RA. Current treatment strategies for age-related ocular conditions. Am J Manag Care. 2013;19(Suppl 5):S76-84.

10. Gray TA, Fenerty C, Harper R, Spencer AF, Campbell M, Henson DB, et al. Individualised patient care as an adjunct to standard care for promoting adherence to ocular hypotensive therapy: an exploratory randomised controlled trial. Eye (Lond). 2012;26:40717. doi:10.1038/eye.2011.269

11. Crews JE, Jones GC, Kim JH. Double jeopardy: the effects of comorbid conditions among older people with vision loss. J Vis Impair Blind. 2006. doi:10.1177/0145482X0610001S07. [Consultada el 29 de Diciembre de 2019].

12. Court H, McLean G, Guthrie B, Mercer SW, Smith DJ. Visual impairment is associated with physical and mental comorbidities in older adults: a cross-sectional study. BMC Med. 2014;12:181. doi:10.1186/s12916-014-0181-7

13. Moyer VA; U.S. Preventive Services Task Force. Screening for glaucoma: U.S. Preventive Services Task Force Recommendation Statement. Ann Intern Med. 2013;159:484-9. doi:10.7326/0003-4819-159-6-2013 09170-00686

14. Jiménez-Ramírez F, Pérez R. Diabetic retinopathy education and screening at the community pharmacy in Puerto Rico. P R Health Sci J. 2011;30:139-44.

15. Berenguer B, La Casa C, de la Matta MJ, Martín-Calero MJ. Pharmaceutical care: past, present and future. Curr Pharm Des. 2004;10:3931-46. doi:10. 2174/1381612043382521

16. Chisholm-Burns MA, Kim Lee J, Spivey CA, Slack M, Herrier RN, Hall-Lipsy E, et al. US pharmacists' effect as team members on patient care: systematic review and meta-analyses. Med Care. 2010;48:923-33. doi: 10.1097/MLR.0b013e3181e57962

17. Marques LA, Galduróz JC, Fernandes MR, Oliveira CC, Beijo LA, Noto AR. Assessment of the effectiveness of pharmacotherapy follow-up in patients treated for depression. J Manag Care Pharm. 2013;19:218-27. doi:10.18553/jmcp.2013.19.3.218

18. Martínez Martínez F, Farragher T, Faus Dáder MJ, García Cárdenas MV, Gastelurrutia Garralda MA, Jódar Sánchez F, et al. Medida del impacto clínico, económico y humanístico del servicio del seguimiento farmacoterapéutico en mayores polimedicados, en la farmacia comunitaria española. 1st ed. ISBN: 978-84-87276-83-5. Madrid: Consejo General de Colegios Oficiales de Farmacéuticos; 2014.

19. Rollason V, Vogt N. Reduction of polypharmacy in the elderly: a systematic review of the role of the pharmacist. Drugs Aging. 2003; 20:817-32. doi:10.2165/00002512-200320110-00003

20. Bjerrum L, Rosholm JU, Hallas J, Kragstrup J. Methods for estimating the occurrence of polypharmacy by means of a prescription database. Eur J Clin Pharmacol. 1997;53:7-11. doi:10.1007/s002280050329

21. Sabater Hernández D, Silva Castro MM, Faus Dader MJ. Dader method. Medication review with follow-up guidelines. 3rd ed. Granada: Pharmaceutical Care Research Group; 2007. Disponible en: https://www. ugr.es/ cts 131/esp/guias/GUIA\%20 FINAL\%20DADER.pdf. [Consultada el 29 de Diciembre de 2019].

22. Knobel H, Alonso J, Casado JL, Collazos J, González J, Ruiz I, et al. Validation of a simplified medication adherence questionnaire in a large cohort of HIV-infected patients: the GEEMA Study. AIDS.
2002;16:605-13. doi:10.1097/00002030200203080-00012

23. García Delgado P, Gastelurrutia Garralda MA, Baena Parejo MI, Fisac Lozano F, Martínez Martínez F. Validation of a questionnaire to assess patient knowledge of their medicines. Aten Primaria. 2009;41:661-8. doi:10.1016/j.aprim.2009.03.011

24. The Royal College of Ophthalmologists (Scientific Department). Cataract Surgery Guidelines September 2010. Disponible en: https:// www.rcophth.ac.uk/wp-content/ uploads/2014/12/2010-SCI-069-Cataract-Surgery-Guidelines-2010-SEPTEMBER-2010-1.pdf. [Consultada el 29 de Diciembre de 2019].

25. Augustin AJ, Offermann I, Lutz J, Schmidt-Erfurth U, Tornambe P. Comparison of the original Amsler grid with the modified Amsler grid: result for patients with age-related macular degeneration. Retina. 2005;25:443-5. doi:10.1097/00006982-20050600000008

26. Shah S, Murdoch IE. NICE - impact on glaucoma case detection. Ophthalmic Physiol Opt. 2011; 31:339-42. doi:10.1111/j.1475-1313.2011.00843.x

27. Research in dry eye: report of the Research Subcommittee of the International Dry Eye WorkShop (2007). Ocul Surf. 2007;5:179-93. doi:10.1016/ S1542-0124(12)70086-1

28. Begley CG, Chalmers RL, Abetz L, Venkataraman K, Mertzanis P, Caffery $\mathrm{BA}$, et al. The relationship between habitual patient-reported symptoms and clinical signs among patients with dry eye of varying severity. Invest $0 \mathrm{ph}$ thalmol Vis Sci. 2003;44:4753-61. doi:10.1167/iovs.03-0270

29. Li J, Tripathi RC, Tripathi BJ. Drug-induced ocular disorders. Drug Saf. 2008;31:127-41. doi:10.2 165/00002018200831020-00003

30. Raizman MB, Hamrah P, Holland EJ, Kim T, Mah FS, Rapuano CJ et al. Drug-induced corneal epithelial changes. Surv Ophthalmol. 2017; 62:286-301. doi:10.1016/j.survophthal. 2016. 11.008 